\title{
Microstructure Imaging Sequence Simulation Toolbox
}

\author{
Andrada Ianuş ${ }^{1}$, Daniel C. Alexander ${ }^{1}$, and Ivana Drobnjak ${ }^{1}$ \\ Centre for Medical Image Computing, Dept. of Computer Science, \\ University College London, UK
}

\begin{abstract}
This work describes Microstructure Imaging Sequence Simulation Toolbox (MISST), a practical diffusion MRI simulator for development, testing, and optimisation of novel MR pulse sequences for microstructure imaging. Diffusion MRI measures molecular displacement at microscopic level and provides a non-invasive tool for probing tissue microstructure. The measured signal is determined by various cellular features such as size, shape, intracellular volume fraction, orientation, etc., as well as the acquisition parameters of the diffusion sequence. $\mathrm{Nu}-$ merical simulations are a key step in understanding the effect of various parameters on the measured signal, which is important when developing new techniques for characterizing tissue microstructure using diffusion MRI. Here we present MISST - a semi-analytical simulation software, which is based on a matrix method approach and computes diffusion signal for fully general, user specified pulse sequences and tissue models. Its key purpose is to provide a deep understanding of the restricted diffusion MRI signal for a wide range of realistic, fully flexible scanner acquisition protocols, in practical computational time.
\end{abstract}

\section{Introduction}

Diffusion MRI (dMRI) has become one of the most important imaging modalities to probe tissue microstructure with many applications in biomedical imaging [12. The dMRI signal measures the displacement of the water molecules inside the tissue and is sensitive to the configuration of cellular membranes, therefore it provides relevant information for characterising tissue properties at the micron level. By developing geometrical models of the tissue and relating them to the acquired dMRI data, it is possible to estimate cellular features such as size, shape, volume fraction, dominant orientation etc. Such estimates provide valuable biomarkers for studying the brain structure or for diagnosing and monitoring diseases. In order to develop fast and reliable acquisition protocols, a good understanding of the most relevant tissue features which affect the dMRI signal as well the influence of different acquisition parameters is very important.

Numerical simulations provide a cheap and powerful tool to investigate the effect of various sequence parameters and tissue features on the measured signal. With synthetic data, we can investigate the ability of various imaging techniques, such as AxCaliber [3], ActiveAx 4], VERDICT [5], to estimate microstructural 
parameters from the data. We can also analyse the effect of including additional tissue features in the model, e.g. fibre dispersion [6] or size distribution [7, of varying acquisition parameters [8] or introducing novel diffusion sequences [9], in a controlled way with known ground truth.

Diffusion MR data synthesis can be divided into three broad categories:

Analytical models have a closed form solution which approximate the diffusion process in bounded geometries under various assumptions. Such approaches include the Short Pulse Gradient (SGP) approximation [10] or the Gaussian Phase Distribution (GPD) approximation [11]12[13]. The signal is fast to compute, however it departs from ground truth values when the assumptions are broken and cannot recover some signal features such as diffusion-diffraction patterns.

Semi-analytical models are based on matrix operators to calculate the time evolution of the diffusion signal inside simple geometries. Such approaches include the matrix formalism introduced by Callaghan [14 or the Multiple Correlation Function (MCF) technique [15]. The diffusion signal can be computed for arbitrary gradient waveforms, is accurate and relatively fast to compute, however, these techniques can be used only for simple geometries with known solutions of the diffusion equation (parallel planes, cylinders, spheres [11, spherical shells [12, triangles [16]).

Numerical models simulate the diffusion process either by numerical solutions of the diffusion equation in a known substrate (e.g. 17, [18) or using a MonteCarlo approach (e.g. 19, 20]). Such techniques can represent more complex and realistic diffusion substrates, however, they are computationally demanding and do not provide the same level of mathematical insight as analytical models do.

Analytical approximations of the dMRI signal are fast to compute but not very accurate, while numerical simulations have higher accuracy and can represent complicated geometries but require significantly more computational power. In simple geometries, semi-analytical approaches represent the middle ground, providing accurate signal calculations in a short computational time.

In this work we present the MISST software package, which is based on a semi-analytical approach, namely the 3D extension 21] of the matrix method proposed by Callaghan. MISST simulates the diffusion MRI signal for generalized, user defined, gradient waveforms and a variety of diffusion substrates. The matrix method has been used so far in various research studies to help validate the GPD approximation for oscillating gradients [13, to analyze the sensitivity of pulsed and oscillating gradients to axon diameter [8] as well as to investigate the properties of a newly introduced diffusion sequence, namely double oscillating diffusion encoding [9]. MISST combines a powerful method for simulating diffusion MRI signal with a wide range of diffusion substrates in a flexible, user friendly software package.

\section{Theory}

This section presents the theory behind the building blocks of MISST. 


\subsection{Diffusion contrast}

Diffusion MRI contrast is obtained by applying a magnetic field gradient with zero first moment at echo time, i.e. $\int_{0}^{T E} \mathbf{G}(t) \cdot d t$, where $\mathbf{G}$ denotes the effective gradient after accounting for the effect of inversion pulses from the imaging sequence. Thus, the phase acquired by each spin is $\phi(t)=\gamma \int_{0}^{t} \mathbf{G}\left(t^{\prime}\right) \cdot \mathbf{r}\left(t^{\prime}\right) d t^{\prime}$ and the measured diffusion signal decay of the spin ensemble $E=\langle\exp (i \phi)\rangle$.

In case of free (Gaussian) diffusion and gradient with a fixed orientation for each measurement, the signal has the well known form:

$$
E=\exp (-b D), \quad \text { where } b=\gamma^{2} \int_{0}^{T E}\left|\int_{0}^{t} \mathbf{G}\left(t^{\prime}\right) d t^{\prime}\right|^{2} d t .
$$

For a generalized gradient waveform, equation 1 needs to be expanded to a tensor form [22]:

$$
E=\exp (-<\mathbf{B}, \mathbf{D}>), \quad \text { where } \mathbf{B}=\gamma^{2} \int_{0}^{T E} \mathbf{F}(t) \mathbf{F}^{T}(t) d t, \mathbf{F}(t)=\int_{0}^{t} \mathbf{G}\left(t^{\prime}\right) d t^{\prime},
$$

$<,>$ denotes the tensor inner product and $\mathbf{D}$ is the diffusion tensor.

In case of diffusion restricted within closed pores, the solution is not straightforward and the signal depends on the propagator $P\left(\mathbf{r}_{0}, t_{0} \mid \mathbf{r}_{1}, t_{1}\right)$ which represents the probability that a particle moves from position $\mathbf{r}_{0}$ at time $t_{0}$ to position $\mathbf{r}_{1}$ at time $t_{1}[23$.

Matrix formalism To simulate restricted diffusion, MISST uses the 3D extension of the matrix method (MM) 1424, which is based on a multiple propagator approach [25. MM provides a generic framework for evaluating the restricted diffusion signal $E$ in a closed form under generalised gradient waveforms. The pulse sequence is divided into narrow intervals $\tau$, as illustrated in Fig. 1 and the gradient amplitude $g_{0}(k \tau)$ is discretized into steps of size $g_{\text {step }}$. Thus, at time $k \tau$ the amplitude of the diffusion vector is $m_{k} q$ where $q=(2 \pi)^{-1} \tau g_{\text {step }}$, and $m_{k}=\left\lfloor\left(g_{0}(k \tau) / g_{\text {step }}\right)\right\rfloor$.

The signal $E$ is calculated as a product of matrix operators which describe the phase evolution inside the boundaries:

$$
E=S(\mathbf{q}) R[A(\mathbf{q})]^{m_{2}} R \ldots R[A(\mathbf{q})]^{m_{K-1}} R S^{T}(-\mathbf{q}),
$$

where the elements of the matrices S, A and R have the following definitions

$$
\begin{aligned}
S_{n}(\mathbf{q}) & =V^{-1 / 2} \int u_{n}(\mathbf{r}) \exp (i 2 \pi \mathbf{q} \cdot \mathbf{r}) d \mathbf{r}, \\
R_{n n} & =\exp \left(-\lambda_{n} D \tau\right), \\
A_{n n^{\prime}}(\mathbf{q}) & =\int u_{n}^{*}(\mathbf{r}) u_{n^{\prime}}(\mathbf{r}) \exp (i 2 \pi \mathbf{q} \cdot \mathbf{r}) d \mathbf{r}
\end{aligned}
$$




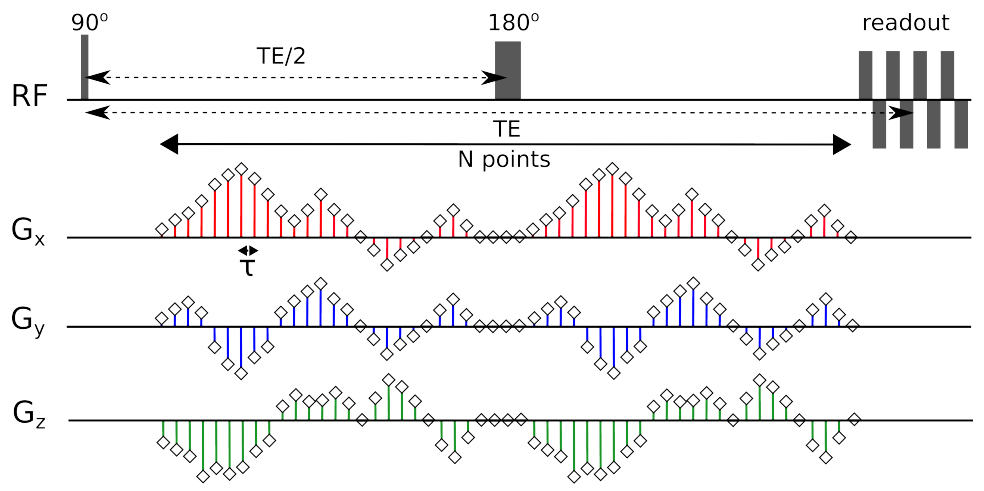

Fig. 1: Schematic representation of a generalized waveform which is repeated before and after the refocusing pulse in a spin-echo sequence. The gradient waveform along each direction is discretized in $\mathrm{K}$ steps.

$V$ is the pore volume, $\mathbf{q}=q \hat{\mathbf{g}}$ where $\hat{\mathbf{g}}$ is the unit gradient vector and $u_{n}$ and $\lambda_{n}$ are the eigenfunctions and eigenvalues of the diffusion equation in the given geometry.

The above method has recently been extended for gradients with time varying orientation 24]. In the case of fixed orientation, the vector $\mathbf{q}$ is the same at every time point $k \tau$ which allows the precalculation of matrices $A(\mathbf{q})$ and $S(\mathbf{q})$. However, when the gradient orientation is time-dependant, the vector $\mathbf{q}$ is different at different time points and the matrices also depend on time. Thus equation 3 becomes:

$$
E=S\left(q \hat{\mathbf{g}}_{\mathbf{1}}\right) R\left[A\left(q \hat{\mathbf{g}}_{\mathbf{2}}\right)\right]^{m_{2}} R \ldots R\left[A\left(q \hat{\mathbf{g}}_{\mathbf{K}-\mathbf{1}}\right)\right]^{m_{K}-1} R S^{T}\left(-q \hat{\mathbf{g}}_{\mathbf{N}}\right),
$$

where $\hat{\mathbf{g}}_{\mathbf{k}}$ denotes the gradient orientation at time $k \tau$.

Calculating the matrices $A\left(q \hat{\mathbf{g}}_{\mathbf{k}}\right)$ element-by-element at each time point is too computationally expensive. To decrease computational time, MISST uses an efficient implementation based on the Taylor expansion of the matrices, which has been proposed and validated in [24].

\subsection{Tissue models}

MISST simulates the diffusion signal from a variety of multi-compartment tissue models. Thus, the overall signal is computed as a weighted sum over different compartments:

$$
E=\sum_{n=1}^{n=N} f_{n} E_{n}, \quad \text { with } \sum_{n=1}^{n=N} f_{n}=1,
$$

where $E_{n}$ and $f_{n}$ are the signal and the volume fraction of the $n^{t h}$ compartment and $N$ is the total number of compartments in the model. For compartments exhibiting Gaussian diffusion, the signal is computed according to equation 2 , while for restricted compartments it is calculated according to equation 5. 


\section{Software implementation}

\subsection{General overview}

MISST is implemented in Matlab using a modular design and is schematically represented in Fig. 2. This implementation allows the user to choose between various diffusion sequences which are widely used in the literature and/or to define their own sequences as well as to build a large variety of tissue models by easily combining the diffusion signal from basic compartments. The package is open source and available for download http://mig.cs.ucl.ac.uk/index.php?n=Tutorial.MISST.

\subsection{Implementation details}

In order to generate the diffusion signal, the user inputs the parameters of the diffusion sequences and of the tissue model. The simulator outputs the diffusion signal and, optionally, the Jacobian of the signal.

Input parameters

Diffusion sequences: The diffusion measurements are represented as a structure, commonly denoted as "protocol", which stores the information regarding the effective diffusion gradient $G$ and the time discretization $\tau$. The 3D gradient waveform is specified as a $\mathrm{M} \times 3 \mathrm{~K}$ matrix, where $\mathrm{M}$ is the number of diffusion measurements and $\mathrm{K}$ is the number of gradient points in one measurement along each direction. The gradient does not necessarily need to be repeated after the $180 \mathrm{rf}$ pulse, nevertheless, the gradient integral should be zero. We provide a set of examples how to generate the discrete gradient waveforms for several diffusion sequences which are widely used in the literature: pulsed gradient spin echo sequences (PGSE), sinusoidal/square/trapezoidal oscillating gradients (OGSE), double pulsed field gradients, stimulated echo sequences, etc. A detailed descriptions for the parametrization of each sequence can be found in the software documentation.

Tissue models: For the diffusion substrates, MISST provides a flexible design of multi-compartment tissue models by combining basic building blocks which have different diffusion characteristics. The different basic models are illustrated in Fig. 2 and follow the nomenclature presented in [26. Currently, there are several multi-compartment white matter models available in MISST, nevertheless, other substrates can be easily implemented by combining different compartments. The information related to the diffusion substrate is represented as a structure commonly referred to as "model". The user needs to specify the model name as well as the model parameters such as diffusivity, radius (for cylinders and spheres), volume fractions of different compartments, etc.

\section{Output parameters}

MISST outputs a vector of normalized diffusion signals corresponding to each measurement in the protocol, for the diffusion substrate specified by the tissue model. Optionally, the simulator can output the signal Jacobian, i.e. the derivatives of the signal with respect to the model parameters. For instance, if the protocol has M measurements and the model has $\mathrm{P}$ parameters, the Jacobian is an $\mathrm{M} \times \mathrm{P}$ matrix. 


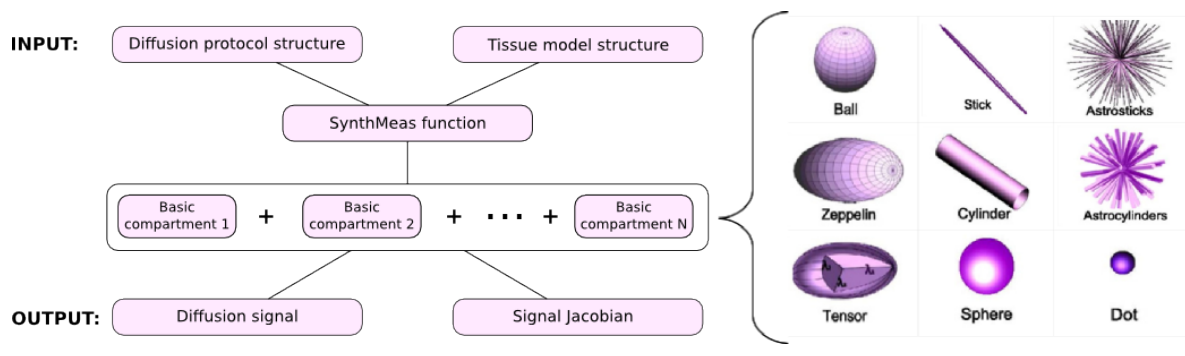

Fig. 2: Schematic representation of MISST, showing the input and output parameters as well as the basic diffusion compartments.

\section{Simulations and results}

\subsection{Signal validation}

The first set of experiments validate the restricted diffusion signal computed from MISST against the Monte Carlo (MC) diffusion simulator in Camino [19] in a substrate consisting of parallel cylinders with radius $R=3 \mu \mathrm{m}$ and intrinsic diffusivity $D=2 \cdot 10^{-9} \mathrm{~m}^{2} / \mathrm{s}$, oriented along the $\mathrm{z}$ axis, as illustrated in Fig. 3 a).

To show the true potential of MISST, in the first simulation we test a protocol with $M=50$ fully generalized gradient waveforms, which are generated by random numbers for gradients in $\mathrm{x}, \mathrm{y}$ and $\mathrm{z}$ directions. To ensure a null gradient integral, the waveforms with a duration of $20 \mathrm{~ms}$ are repeated before and after the $180 \mathrm{rf}$ pulse, as schematically illustrated in Fig. 3 3 ). We set the maximum gradient strength to $500 \mathrm{mT} / \mathrm{m}$ and we use a time step $\tau=0.1 \mathrm{~ms}$. For the MC simulations we used 200000 walkers and the same time step $\tau$. The plot in Fig. 3k) presents the diffusion signal computed using the two methods. The signal difference between MISST and MC is less than $0.16 \%$ for all data points. The computational time necessary for MISST to generate this data set was $5 \mathrm{~s}$, while the MC simulation took 10min.

The second simulation investigates an acquisition protocol consisting of double pulsed field gradient sequences ( $\mathrm{dPFG}$ ) which vary the angle between the gradients in the plane perpendicular to the cylinder axis. A schematic representation of the sequence is illustrated in Fig. $3 \mathrm{~d})$. The sequence parameters for this simulation are: gradient strength $G=500 \mathrm{mT} / \mathrm{m}$, pulse duration $\delta=2 \mathrm{~ms}$, pulse separation $\Delta=50 \mathrm{~ms}$, mixing time $\tau_{m}=\{0,2,10\} \mathrm{ms}$ and angle $\varphi$ between the gradient directions from 0 and $2 \pi$. The plots in Fig. 3 e) show the dependence of the dPGSE signal as a function of the angle between the gradients for three different mixing times, when the signal was generated either using MISST or MC simulations. Similarly to the previous simulations, the signal difference between the two methods is less than $0.3 \%$ for all the data points. In this case, MISST calculations were performed in 10 s, while the MC simulation took $1 \mathrm{~h} 20 \mathrm{~min}$.

The third analysis shows that MISST can reproduce important features of the restricted diffusion signal which cannot be accurately modelled with simpler ana- 


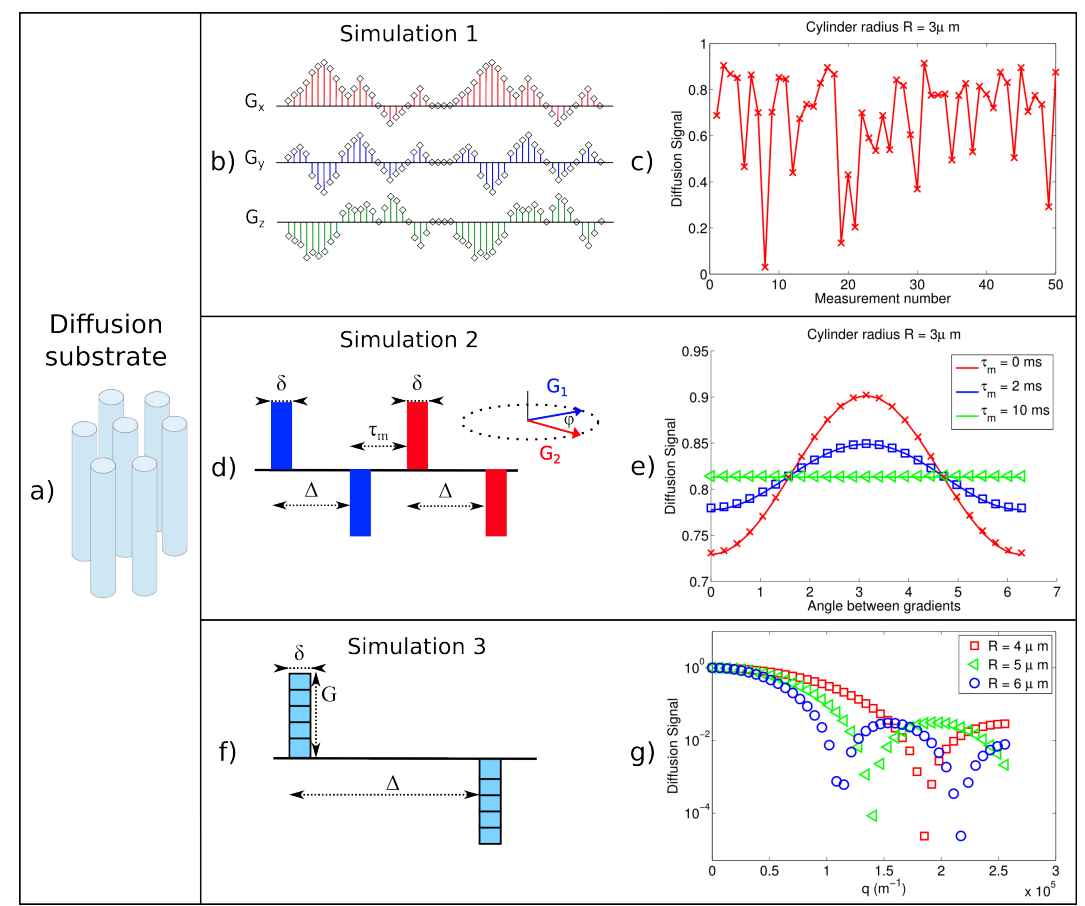

Fig. 3: a) Schematic representation of the diffusion substrate; Schematic representation of b) the random gradient waveform and c) the corresponding diffusion signals obtained using MISST (line) and MC simulations (symbol); Schematic representation of d) the dPGSE sequence and e) the corresponding diffusion signals obtained using MISST (line) and MC simulations (symbol); Schematic representation of $\mathrm{f}$ ) the PGSE sequence and $\mathrm{g}$ ) the corresponding diffusion-diffraction patterns obtained using MISST.

lytical approximations like GPD, such as diffusion-diffraction patterns when the wave vector $q=(2 \pi)^{-1} \gamma G \delta$ is increased 2728 . In this simulation we use standard PGSE sequences with $\delta=3 \mathrm{~ms}, \Delta=100 \mathrm{~ms}$ and $G=\{0,50,100, \ldots, 2000\}$ $\mathrm{mT} / \mathrm{m}$. The diffusion-diffraction patterns depend on the restriction size, as illustrated in Fig. 3s) for parallel cylinders with three different radii $R=\{4,5,6\} \mu \mathrm{m}$.

\subsection{Application example}

MISST software can be used for a wide range of applications, from validating analytical approximations [13, to analysing the sensitivity of various diffusion measurements [8] or understanding the contrast of novel sequences [9]. Here we illustrate an example of using MISST to compare the sensitivity of PGSE and OGSE sequences to pore diameter in cylindrical restriction. We investigate two situations: 1) the gradient is orthogonal to the cylinder axis and 2) the gradient is not perfectly perpendicular, deviating by a small angle $\theta=10^{\circ}$. We analyse a 


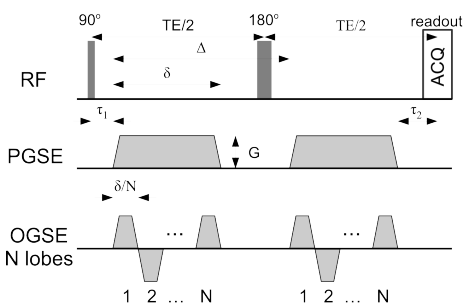

a)

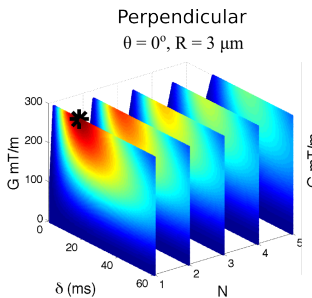

$\delta$ (ms)

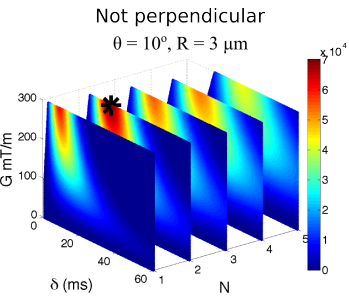

b)

Fig. 4: Sensitivity of PGSE $(\mathrm{N}=1)$ and OGSE $(\mathrm{N}>1)$ sequences to cylinder radius for a wide range of sequence parameters. The star denotes the most sensitive sequence.

wide range of practical sequence parameters with gradient strength $G \in[0,300]$ $\mathrm{mT} / \mathrm{m}, \delta \in[0,60] \mathrm{ms}, \Delta=\delta+10 \mathrm{~ms}$ and $N=\{1,2, \ldots, 5\}$ gradient lobes, as illustrated in Fig. 4 4 ). In this simulation we use a two-compartment tissue model with parallel cylinders $\left(R=3 \mu \mathrm{m}, D=1.7 \cdot 10^{-9} \mathrm{~m}^{2} / \mathrm{s}\right.$, volume fraction $\left.f=0.7\right)$ and hindered extracellular space in the tortuosity limit $\left(D_{\|}=1.7 \cdot 10^{-9} \mathrm{~m}^{2} / \mathrm{s}\right.$, $\left.D_{\perp}=(1-f) \cdot D_{\|}\right)$. We account for the effect of T2 decay with a constant $T 2=70 \mathrm{~ms}$. An in-depth analysis of PGSE vs. OGSE sensitivity to pore diameter is presented in 8 .

Figure $4 \mathrm{p})$ plots the sensitivity of PGSE $(\mathrm{N}=1)$ and OGSE $(\mathrm{N}>1)$ sequences with a wide range of parameters. The results show that in the case when the gradient direction is orthogonal to the cylinder orientation, PGSE sequences have the highest sensitivity, however, if the gradient is no longer perfectly perpendicular or there is fibre dispersion (not shown here), OGSE sequences with low frequency yield the highest sensitivity. This analysis shows the importance of numerical simulations for exploring optimal combinations of sequence parameters in an intuitive way.

\section{Discussion}

This work introduces MISST, a software package that simulates the diffusion MRI signal from a variety of pulses sequences and diffusion substrates. Being open source, the user can easily tailor the software to explore their own research question, allowing faster development in the field of diffusion MRI.

MISST implements the 3D extension of the matrix method, which allows the computation of restricted diffusion signal for flexible, user defined, gradient waveforms. We provide details of the implementation, as well as examples of tissue models and gradient waveforms. The signal calculation is accurate and preserves important signal features such as diffusion-diffraction patterns, yet it is orders of magnitude faster to compute compared to MC simulations, which makes it practical for many applications.

One limitation of the matrix method is that it can be used to calculate the restricted diffusion signal only for basic geometries with well known solution of 
the diffusion equation such as parallel planes, cylinders, spheres, spherical shells as well as triangles. Another limitation is the fact that diffusion in extracellular space needs to be computed separately, thus the accuracy of the signal depends on the complexity of the chosen model. Although boundary relaxation effects can be accounted for in the matrix method formalism, the exchange between intra and extracellular spaces cannot be readily incorporated.

Future work aims to provide the template for more diffusion sequences which have been recently developed in the literature (double oscillating diffusion encoding, q-mas and other sequences with isotropic encoding) as well as more diffusion compartments such as cuboids and finite cylinders [7.

The novelty of MISST is that it simulates dMRI signal for any user-defined diffusion gradient waveform, from a standard PGSE to more advanced sequences which are of great interest to the research in this field. Moreover, due to its modular construction, the user can easily combine various diffusion compartments to create models that are representative of various tissue types, such as grey matter, white matter or tumours. All these features are combined in a user-friendly, open source software package.

\section{References}

1. D. K. Jones. Diffusion MRI: theory, methods and application. Oxford University Press, 2010.

2. H. Johansen-Berg and T. E. J. Behrens. Diffusion MRI: from quantitative measurement to in vivo neuroanatomy. Academic Press, 2009.

3. Y. Assaf, T. Blumenfeld-Katzir, Y. Yovel, and P. J. Basser. AxCaliber: a method for measuring axon diameter distribution from diffusion MRI. Magnetic Resonance in Medicine, 59:1347-1354, 2008.

4. D. C. Alexander, P. L. Hubbard, M. G. Hall, E. A. Moore, M. Ptito, G. J. M. Parker, and T. B. Dyrby. Orientationally invariant indices of axon diameter and density from diffusion MRI. NeuroImage, 52:1374-1389, 2010.

5. E. Panagiotaki, S. Walker-Samuel, B. Siow, S. P. Johnson, V. Rajkumar, R. B. Pedley, M. F. Lythgoe, and D. C. Alexander. Noninvasive quantification of solid tumor microstructure using VERDICT MRI. Cancer Research, 74:1902-1912, 2014.

6. H. Zhang, P. L. Hubbard, G. J. M. Parker, and D. C. Alexander. Axon diameter mapping in the presence of orientation dispersion with diffusion MRI. NeuroImage, 56:1301-1315, 2011.

7. A. Ianuş, I. Drobnjak, and D. C. Alexander. Model-based estimation of microscopic anisotropy using diffusion MRI: a simulation study. NMR in Biomedicine, 29:627$685,2016$.

8. I. Drobnjak, H. Zhang, A. Ianuş, E. Kaden, and D. C. Alexander. PGSE, OGSE, and sensitivity to axon diameter in diffusion MRI: Insight from a simulation study. Magnetic Resonance in Medicine, 75:688-700, 2016.

9. N. Shemesh, A. Ianuş, D. C. Alexander, and I. Drobnjak. Double oscillating diffusion encoding (dode) augments microscopic anisotropy contrast. In Proc. ISMRM, page 952, Toronto, Canada, 2015.

10. E. O. Stejskal. Use of spin echoes in a pulsed magnetic-field gradient to study anisotropic, restricted diffusion and flow. The Journal of Chemical Physics, 43:3597-3603, 1965. 
11. C. H. Neuman. Spin echo of spins diffusing in a bounded medium. Journal of Chemical Physics, 60:4508-4511, 1974.

12. J. Xu, M. D. Does, and J. C. Gore. Quantitative characterization of tissue microstructure with temporal diffusion spectroscopy. Journal of Magnetic Resonance, 200:189-197, 2009.

13. A. Ianuş, B. Siow, I. Drobnjak, H. Zhang, and D. C. Alexander. Gaussian phase distribution approximations for oscillating gradient spin-echo diffusion (MRI). Journal of Magnetic Resonance, 227:25-34, 2013.

14. P. T. Callaghan. A simple matrix formalism for spin echo analysis of restricted diffusion under generalized gradient waveforms. Journal of Magnetic Resonance, 129:74-84, 1997.

15. D. Grebenkov. Laplacian eigenfunctions in NMR. i. a numerical tool. Concepts in Magnetic Resonance Part A, 32:277-301, 2008.

16. T. A. Kuder and F. B. Laun. NMR-based diffusion pore imaging by double wave vector measurements. Magnetic Resonance in Medicine, 70:836-841, 2013.

17. J. Xu, M. D. Does, and J. C. Gore. Numerical study of water diffusion in biological tissues using an improved finite difference method. Physics in Medicine and Biology, 52:N111-N126, 2007.

18. J-R. Li, D. Calhoun, C. Poupon, and D. Le Bihan. Numerical simulation of diffusion mri signals using an adaptive time-stepping method. Physics in Medicine and Biology, 59:441-454, 2014.

19. M. G. Hall and D. C. Alexander. Convergence and parameter choice for MonteCarlo simulations of diffusion MRI. IEEE Trans. Medical Imaging, 28:1354-1364, 2009.

20. C. H. Yeh, B. Schmitt, D. Le Bihan, J. R. Li-Schlittgen, C. P. Lin, and C. Poupon. Diffusion microscopist simulator: A general monte carlo simulation system for diffusion magnetic resonance imaging. PLoS ONE, 2013.

21. I. Drobnjak and D. C. Alexander. Optimising time-varying gradient orientation for microstructure sensitivity in diffusion-weighted MR. Journal of Magnetic Resonance, 212:344-354, 2011.

22. C. F. Westin, F. Szczepankiewicz, O. Pasternak, E. Özarslan, D. Topgaard, H. Knutsson, and M. Nilsson. Measurement tensors in diffusion mri: Generalizing the concept of diffusion encoding. In Proc. MICCAI, pages 209-216, Boston, 2014. Springer.

23. P. T. Callaghan. Principles of Magnetic Resonance Microscopy. Oxford, UK: Oxford Science Publications, 1991.

24. I. Drobnjak, H. Zhang, M. G. Hall, and D. C. Alexander. The matrix formalism for generalised gradients with time-varying orientation in diffusion NMR. Journal of Magnetic Resonance, 210:151-157, 2011.

25. A. Caprihan, L. Z. Wang, and E. Fukushima. A multiple-narrow-pulse approximation for restricted diffusion in a time-varying field gradient. Journal of Magnetic Resonance, Series A, 118:94-102, 1996.

26. E. Panagiotaki, T. Schneider, B. Siow, M. G. Hall, M. F. Lythgoe, and D. C. Alexander. Compartment models of the diffuison MR signal in brain white matter: a taxonomy and comparison. NeuroImage, 59:2241-2254, 2012.

27. P. T. Callaghan, A. Coy, D. MacGowan, K. J. Packer, and F. O. Zelaya. Diffractionlike effects in NMR diffusion studies of fluids in porous solids. Nature, 351:467-469, 1991.

28. B. Balinov, B. Jonsson, P. Linse, and O. Soderman. The NMR self-diffusion method applied to restricted diffusion. Simulation of echo attenuation from molecules in spheres and between planes. Journal of Magnetic Resonance., 104:17-25, 1993. 